EESTI NSV TEADUSTE AKADEEMIA TOIMETISED. XII KÖIDE

FUUSIKA-MATEMAATIKA- JA TEHNIKATEADUSTE SEERIA. 1963, NR. 2

ИЗВЕСТИЯ АКАДЕМИИ НАУК ЭСТОНСКОИ ССР. ТОМ ХІІ СЕРИЯ ФИЗИКО-МАТЕМАТИЧЕСКИХ И ТЕХНИЧЕСКИХ НАУК. 1963, № 2

\title{
ЭКСТРЕМАЛЬНАЯ ЗАДАЧА ДЛЯ ОДНОГО КЛАССА ФУНКЦИй
}

\section{М. ЛЕВИН}

Пусть множество $F(M)$ состоит из всех функций $f(x, y)$, удовлетворяющих на $[0,1 ; 0,1]$ условиям: $f(x, 0) \equiv f(0, y) \equiv 0 ; f_{x}^{\prime}, f_{y}^{\prime}$ и $f_{x y}^{\prime \prime}$ ограничены, интегрируемы и

$$
\left\{\int_{0}^{1} \int_{0}^{1}\left[f_{x y}^{\prime \prime}(x, y)\right]^{2} d x d y\right\}^{1 / 9} \leqslant M
$$

Аналогично тому, как это обычно делается [1, 2$]$, построим для этого множества функций наилучшую кубатурную формулу вида

$$
\int_{0}^{1} \int_{0}^{1} f(x, y) d x d y=\sum_{i, j=0}^{m-1, n-1} A_{i j} f\left(x_{i}, y_{j}\right)+R(f) .
$$

Для этого надо выбрать числа $A_{i j}, x_{i}, y_{i}(i=0,1, \ldots, m-1$; $j=0,1, \ldots, n-1)$ так, чтобы величина

$$
R=\sup _{f \in F(M)}|R(f)|
$$

была наименьшей.

Функцию $f(x, y) \in F(M)$ можно записать в виде

$$
f(x, y)=\int_{0}^{1} \int_{0}^{1} f_{x y}^{\prime \prime}(t, u) E(x-t) E(y-u) d t d u
$$

где

$$
E(x)= \begin{cases}0, & x \leqslant 0 \\ 1, & x>0\end{cases}
$$

По (1), используя (2) и неравенство Буняковского, имеем оценку ошибки формулы (1) в виде

$$
|R(f)| \leqslant M\left\{\int_{0}^{1} \int_{0}^{1}[K(t, u)]^{2} d t d u\right\}^{1 / 2}
$$

где

$$
K(t, u)=(1-t)(1-u)-\sum_{i, j=0}^{m-1, n-1} A_{i j} E\left(x_{i}-t\right) E\left(y_{j}-u\right)
$$


Так как для функции

$$
f(x, y)=\frac{M \int_{0}^{x} \int_{0}^{y} K(t, u) d t d u}{\left[\int_{0}^{1} \int_{0}^{1}|K(t, u)|^{2} d t d u\right]^{1 / 2}}
$$

неравенство (3) превращается в равенство, то

$$
\sup _{f \in F(M)} R(f) \mid=M\left\{\int_{0}^{1} \int_{0}^{1}[K(t, u)]^{2} d t d u\right\}^{1 / 2} .
$$

Таким образом, задача свелась к минимизации правой части равенства (4), или, что то же, к минимизации величины

$U=\int_{0}^{1} \int_{0}^{1}[K(t, u)]^{2} d t d u=\int_{0}^{1} \int_{0}^{1}\left[x y-\sum_{i, j=0}^{m-1, n-1} \lambda_{i j} E\left(x-u_{i}\right) E\left(y-v_{j}\right)\right]^{2} d x d y$, где $u_{i}=1-x_{m-i-1}, v_{j}=1-y_{n-j-1}, \lambda_{i j}=A_{m-i-1, n-j-1}$ $(i=0,1, \ldots, m-1 ; j=0,1, \ldots, n-1)$.

Запишем величину $U$ в виде

$$
U=\int_{0}^{u_{0}} \int_{0}^{1} x^{2} y^{2} d x d y+\int_{u_{0}}^{1} \int_{0}^{v_{0}} x^{2} y^{2} d x d y+
$$

$+\int_{u_{0}}^{1} \int_{v_{0}}^{1}\left[x y-\sum_{i, j=0}^{m-1, n-1} \lambda_{i j} E\left(x-u_{i}\right) E\left(y-v_{i}\right)\right]^{2} d x d y=\frac{1}{9}\left(u_{0}^{3}+v_{0}^{3}-u_{0}^{3} v_{0}^{3}\right)+$ $+\sum_{i, j=0}^{m-1, n-1} \int_{u_{i}}^{u_{i}+1} \int_{v_{j}}^{v_{j+1}}\left(x y-c_{i j}\right)^{2} d x d y$

где

$$
\begin{aligned}
c_{i j} & =\sum_{s=0}^{i} \sum_{q=0}^{j} \lambda_{s q} \quad\left(\begin{array}{l}
i=0,1, \ldots, m-1 \\
j=0,1, \ldots, m-1
\end{array}\right), \\
u_{m} & =\tau_{n}=1 .
\end{aligned}
$$

Найдем

$$
\min _{\alpha} \int_{a}^{b} \int_{c}^{d}(x y-\alpha)^{2} d x d y
$$

$$
\begin{aligned}
I=\int_{a}^{b} \int_{c}^{d}(x y-\alpha)^{2} d x d y= & \frac{1}{9}\left(b^{3}-a^{3}\right)\left(d^{3}-c^{3}\right)-\frac{\alpha}{2}\left(b^{2}-a^{2}\right)\left(d^{2}-c^{2}\right)+ \\
& +\alpha^{2}(b-a)(d-c) .
\end{aligned}
$$

Приравнивая $I_{\alpha}^{\prime}$ к нулю, находим

$$
\alpha=\frac{(a+b)(c+d)}{4},
$$


что дает

$$
\begin{gathered}
\min _{\alpha} \int_{a}^{b} \int_{c}^{d}(x y-a)^{2} d x d y=\int_{a}^{b} \int_{c}^{d}\left[x y-\frac{1}{4}(a+b)(c+d)\right]^{2} d x d y= \\
=\frac{1}{9}\left(b^{3}-a^{3}\right)\left(d^{3}-c^{3}\right)-\frac{1}{16}(a+b)\left(b^{2}-a^{2}\right)(c+d)\left(d^{2}-c^{2}\right) .
\end{gathered}
$$

Учитывая это, получим

$$
\begin{aligned}
U \geqslant \frac{1}{9} & \left(u_{0}^{3}+v_{0}^{3}-u_{0}^{3} v_{0}^{3}\right)+\sum_{i=0}^{m-1,} \int_{u_{i}}^{n-1} \int_{v_{j}}^{u_{i}+1}\left[x y-\frac{\left(u_{i}+u_{i+1}\right)\left(v_{j}+v_{j+1}\right)}{4}\right]^{2} d x d y= \\
= & \frac{1}{9}\left(u_{0}^{3}+v_{0}^{3}-u_{0}^{3} v_{0}^{3}\right)+\sum_{i, j=0}^{m-1, n-1}\left[\frac{1}{9}\left(u_{i+1}^{3}-u_{i}^{3}\right)\left(v_{j+1}^{3}-v_{j}^{3}\right)-\right. \\
& \left.-\frac{1}{16}\left(u_{i}+u_{i+1}\right)\left(u_{i+1}^{2}-u_{i}^{2}\right)\left(v_{j}+v_{j+1}\right)\left(v_{j+1}^{2}-v_{j}^{2}\right)\right]= \\
= & -\frac{1}{9} \sum_{i=0}^{m-1}\left(u_{i}+u_{i+1}\right)\left(u_{i+1}^{2}-u_{i}^{2}\right) \sum_{j=0}^{n-1}\left(v_{j}+v_{j+1}\right)\left(v_{j+1}^{2}-v_{j}^{2}\right) .
\end{aligned}
$$

Найдем теперь $u_{i}, v_{j}(i=0,1, \ldots, m-1 ; j=0,1, \ldots, n-1)$, минимизирующие правую часть последнего неравенства. Для этого имеем систему уравнений

$$
\begin{aligned}
& u_{1}^{2}-3 u_{0}^{2}-2 u_{0} u_{1}=0 \\
& u_{2}^{2}-2 u_{1} u_{2}+2 u_{1} u_{0}-u_{0}^{2}=0 \\
& u_{3}^{2}-2 u_{2} u_{3}+2 u_{2} u_{1}-u_{1}^{2}=0 \\
& \cdot \cdot \cdot \cdot \cdot \cdot \cdot \cdot \cdot \cdot \cdot \cdot \cdot u_{m-1} u_{m-2}-u_{m-2}^{2}=0 \\
& u_{m}^{2}-2 u_{m-1} u_{m}+2 u_{m} \\
& u_{m}=1
\end{aligned}
$$

и аналогичную систему для нахождения $v_{j}(j=0,1, \ldots, n)$. Решение этих систем (с учетом условий $u_{i}, v_{j} \geqslant 0, u_{i+1}>u_{i}, v_{j+1}>v_{j}$ ) дает

$$
u_{i}=\frac{2 i+1}{2 m+1}, \quad v_{i}=\frac{2 j+1}{2 n+1} \quad\left(\begin{array}{c}
i=0,1, \ldots, m ; \\
j=0,1, \ldots, n
\end{array}\right)^{*}
$$

Таким образом, величина $U$ достигает своего наименьшего значения при узлах (7). Осталось найти значения $\lambda_{i j}(i=0,1, \ldots, m-1$; $j=0,1, \ldots, n-1)$.

Используя (5), имеем

$$
\sum_{s=0}^{i} \sum_{q=0}^{j} \lambda_{s q}=c_{i i}=\frac{\left(u_{i}+u_{i+1}\right)\left(v_{j}+v_{j+1}\right)}{4} \quad\left(\begin{array}{l}
i=0,1, \ldots, m-1 ; \\
j=0,1, \ldots, n-1
\end{array}\right) .
$$

* То, что именно эти значения минимизируют правую часть неравенства, проверяется обычными методами. 
Подставляя сюда значения (7), имеем

$$
\sum_{s=0}^{i} \sum_{q=0}^{i} \lambda_{s q}=\frac{4(i+1)(j+1)}{(2 m+1)(2 n+1)} \quad\left(\begin{array}{l}
i=0,1, \ldots, m-1 \\
j=0,1, \ldots, n-1
\end{array}\right),
$$

откуда

$$
\lambda_{i j}=\frac{4}{(2 m+1)(2 n+\overline{1})} \quad\left(\begin{array}{c}
i=0,1, \ldots, m-1 ; \\
j=0,1, \ldots, n-1
\end{array}\right)
$$

Используя связь между $x_{i}$ и $u_{i}, y_{j}$ и $v_{j}, A_{i j}$ и $\lambda_{i j}$, находим наилучшую для множества $F(M)$ кубатурную формулу

$$
\int_{0}^{1} \int_{0}^{1} f(x, y) d x d \underline{\approx} \approx \frac{4}{(2 m+1)(2 n+1)} \sum_{i, j=0}^{m-1, n-1} f\left(\frac{2 i+2}{2 m+1}, \frac{2 j+2}{2 n+1}\right) .
$$

Найдем величину максимально возможной ошибки при вычислениях по (8) для функций множества $F(M)$ - значение $R_{0}$ :

$$
\begin{gathered}
R_{0}=\min _{A_{i j}, x_{i}, y_{i} f \in F(M)} \sup _{f \in F(f) \mid=M \sqrt{\min _{A_{i j}, x_{i}, y_{i}} U}=} \mid R\left(\frac{1}{9}-\frac{1}{16} \sum_{i=0}^{m-1} \frac{4(i+1)}{(2 m+1)} \cdot \frac{8(i+1)}{(2 m+1)^{2}} \sum_{j=0}^{n-1} \frac{4(j+1) 8(j+1)}{(2 n+1)^{3}}\right]^{1 / 2}= \\
=\frac{M}{3(2 m+1)(2 n+1)} \sqrt{4\left(m^{2}+n^{2}+m+n\right)+1} .
\end{gathered}
$$

Все изложенное в этой заметке легко обобщается на случай интегралов произвольной кратности.

\section{ЛИТЕРАТ У Р А}

1. С. М. Ни коль ски й, Квадратурные формулы. М., 1958.

2. Ю. Я. Доронин, К вопросу о формулах механических квадратур. Сб. трудов Днепропетровского инж.-стр. ин-та, 1955, 1-2.

Институт кибернетики Академии наук Эстонской ССР
Поступила в редакцию 29. IX 1962 


\title{
EKSTREMAALOLESANNE UHE FUNKTSIOONIDE KLASSI JAOKS
}

\author{
M. Levin \\ Resümee
}

Artiklis lahendatakse kubatuurvalemi (1) jäägi minimiseerimise ülesanne funktsioonide klassi $F(M)$ jaoks, mis koosneb kõigist ruudus $[0,1 ; 0,1]$ tõkestatud, integreeruvaist ning tingimusi $f(x, 0) \equiv f(0, y) \equiv 0$ ja $\left\{\iint_{0}^{1}\left[f_{x y}^{\prime \prime}\right]^{2} d x d y\right\}^{1 / 2} \leqslant M$ rahuldavaist funktsioonidest. Selle ekstremaalülesande lahendiks saadakse kubatuurvalem (8) veahinnanguga (9).

Eesti NSV Teaduste Akadeemia Küberneetika Instituut
Saabus toimetusse

29. IX 1962

\section{A PROBLEM OF MINIMUM FOR ONE CLASS OF FUNCTIONS}

\section{Levin}

\section{Summary}

In this article the problem of finding the minimum of the remainder of formula (1) is solved for the class $F(M)$, which consists of functions $f(x, y) f(x, 0) \equiv f(0, y) \equiv 0$ bounded and integrable on $[0,1 ; 0,1]$

$$
\left\{\int_{0}^{1} \int_{0}^{1}\left[f_{x y}^{\prime \prime}\right]^{2} d x d y\right\}^{1 / 2} \leqslant M
$$

The solution of the problem is given in (8) with the estimation of error in (9).

Academly of Sciences of the Estonian S.S.R., Institute of Cybernetics
Received Sept. 29th, 1962 